\title{
The index of biharmonic maps in spheres
}

\author{
E. Loubeau and C. Oniciuc
}

\begin{abstract}
Biharmonic maps are the critical points of the bienergy functional and generalise harmonic maps. We investigate the index of a class of biharmonic maps derived from minimal Riemannian immersions into spheres. This study is motivated by three families of examples: the totally geodesic inclusion of spheres, the Veronese map and the Clifford torus.
\end{abstract}

\section{Introduction}

In their original 1964 paper [ES64], Eells and Sampson proposed an infinite-dimensional Morse theory on the manifold of smooth maps between Riemannian manifolds. Though their main results concern the Dirichlet energy, they also suggested other functionals. The interest encountered by harmonic maps, and to a lesser extent by $p$-harmonic maps, has overshadowed the study of other possibilities, e.g. exponential harmonicity [EL92]. While the examples mentioned so far are all of first-order functionals, one can investigate problems involving higher-order functionals. A prime example of these is the bienergy, not only for the role it plays in elasticity and hydrodynamics, but also because it can be seen as the next stage of investigation, should the theory of harmonic maps fail.

Witness the case of the two-torus $\mathbb{T}^{2}$ and the two-sphere $\mathbb{S}^{2}$ : Eells and Wood showed in [EW76] that there exists no harmonic map from $\mathbb{T}^{2}$ to $\mathbb{S}^{2}$ (whatever the metrics chosen) in the homotopy classes of Brower degrees \pm 1 . But the situation is drastically different for biharmonicity. By PalaisSmale, in every homotopy class from a surface (or a three-manifold) to a compact manifold, there exists a biharmonic map (cf. [ES66]). While this biharmonic map could very well turn out to be also harmonic, this cannot happen to maps of degrees \pm 1 between $\mathbb{T}^{2}$ and $\mathbb{S}^{2}$.

Let $\phi:(M, g) \rightarrow(N, h)$ be a smooth map between Riemannian manifolds. We define its tension field to be $\tau(\phi)=$ trace $\nabla d \phi$, and, for a compact domain $K \subseteq M$, its bienergy to be

$$
E^{2}(\phi)=\frac{1}{2} \int_{K}|\tau(\phi)|^{2} v_{g}
$$

The critical points of $E^{2}$, with respect to continuous deformations, are called biharmonic maps. The Euler-Lagrange operator associated to $E^{2}$ is

$$
\tau^{2}(\phi)=-\Delta^{\phi}(\tau(\phi))-\operatorname{trace} R^{N}(d \phi, \tau(\phi)) d \phi,
$$

where

$$
\Delta^{\phi}=-\operatorname{trace}_{g}\left(\nabla^{\phi}\right)^{2}=-\operatorname{trace}_{g}\left(\nabla^{\phi} \nabla^{\phi}-\nabla_{\nabla}^{\phi}\right)
$$

is the Laplacian on sections of the pull-back bundle $\phi^{-1} T N$ and $R^{N}$ is the curvature operator

$$
R^{N}(X, Y) Z=\left[\nabla_{X}^{N}, \nabla_{Y}^{N}\right] Z-\nabla_{[X, Y]}^{N} Z, \quad \forall X, Y, Z \in C(T N) .
$$

Hence, a map is biharmonic if and only if $\tau^{2}(\phi)=0$.

Received 16 May 2003, accepted in final form 4 June 2004, published online 21 April 2005.

2000 Mathematics Subject Classification 58E20, 53C42.

Keywords: biharmonic maps, stability.

The first author thanks the Faculty of Mathematics of Iaşi for its hospitality. The second author thanks the CNRS for support.

This journal is (C) Foundation Compositio Mathematica 2005. 


\section{E. Loubeau And C. Oniciuc}

It is interesting to observe that $\tau^{2}(\phi)=-J^{\phi}(\tau(\phi))$, where the Jacobi operator $J^{\phi}$ plays an important role in the theory of harmonic maps, since it gives the second variation of the energy functional $E(\phi)=\frac{1}{2} \int_{M}|d \phi|^{2} v_{g}$ at its critical points, the well-known harmonic maps. Moreover, the Euler-Lagrange operator related to $E$ is precisely the tension field $\tau(\phi)$.

As the Jacobi operator is linear, harmonic maps are trivially biharmonic and, in fact, global minima of $E^{2}$. Besides, by means of a Bochner technique, Jiang established a partial converse, as follows.

Theorem 1.1 [Jia86]. A biharmonic map from a compact domain into a non-positively curved manifold is harmonic.

One can easily construct a non-harmonic biharmonic map, by choosing a third-order polynomial mapping between Euclidean spaces, since, in this situation, the biharmonic operator is nothing but the Laplacian composed with itself.

However, starting from a compact manifold, one must choose positively curved targets, e.g. the sphere. Remembering that, on the one hand, mapping $\mathbb{S}^{n}$ into $\mathbb{S}^{n+1}$ as the equator yields a harmonic map, i.e. a biharmonic map of vanishing bienergy, and that, on the other hand, sending the whole of $\mathbb{S}^{n}$ onto the north pole of $\mathbb{S}^{n+1}$ also has zero bienergy, one can infer the existence of a non-harmonic biharmonic map from $\mathbb{S}^{n}$ into $\mathbb{S}^{n+1}$. A more precise argument (cf. [CMO01]) shows that this map is actually the embedding of $\mathbb{S}^{n}(1 / \sqrt{2})$ in $\mathbb{S}^{n+1}$.

In [CMP01], Caddeo, Montaldo and Piu show that a biharmonic curve on a surface of non-positive Gaussian curvature is a geodesic, i.e. is harmonic, and give examples of non-harmonic biharmonic curves on spheres, ellipses, unduloids and nodoids. On the three-sphere, the only non-harmonic biharmonic curves are certain circles and helices, while $\mathbb{S}^{2}(1 / \sqrt{2})$ is the unique non-harmonic that is a non-minimal, biharmonic surface [CMO01]. More generally, for the sphere $\mathbb{S}^{n}$, only circles and helices are non-harmonic biharmonic curves [CMO02].

Since biharmonicity derives from a variational principle, one is naturally tempted to take the second variation of the functional $E^{2}$. While this was done in general by Jiang [Jia86], in light of the restrictions made in Theorem 1.1 on the curvature of the target space, we will restrict ourselves to maps into the unit sphere. In this configuration, the second variation formula for the bienergy was obtained by the second author, as follows.

Theorem 1.2 [Oni02]. Assume that $(M, g)$ is a compact manifold and $\phi:(M, g) \rightarrow \mathbb{S}^{n}$ is a biharmonic map. Take $\left\{\phi_{s, t}\right\}_{s, t \in \mathbb{R}}$ a two-parameter variation of $\phi$ and set

$$
\left.\frac{\partial \phi_{s, 0}}{\partial s}\right|_{s=0}=V \quad \text { and }\left.\quad \frac{\partial \phi_{0, t}}{\partial t}\right|_{t=0}=W
$$

Then

where

$$
\left.\frac{\partial^{2} E^{2}\left(\phi_{s, t}\right)}{\partial s \partial t}\right|_{(s, t)=(0,0)}=(I(V), W)=\int_{M}\langle I(V), W\rangle v_{g},
$$

$$
\begin{aligned}
I(V)= & \Delta^{\phi}\left(\Delta^{\phi} V\right)+\Delta^{\phi}\left(\operatorname{trace}\langle V, d \phi \cdot\rangle d \phi \cdot-|d \phi|^{2} V\right)+2\langle d \tau(\phi), d \phi\rangle V+|\tau(\phi)|^{2} V \\
& -2 \operatorname{trace}\langle V, d \tau(\phi) \cdot\rangle d \phi \cdot-2 \operatorname{trace}\langle\tau(\phi), d V \cdot\rangle d \phi \cdot-\langle\tau(\phi), V\rangle \tau(\phi)+\operatorname{trace}\left\langle d \phi \cdot, \Delta^{\phi} V\right\rangle d \phi . \\
& +\operatorname{trace}\langle d \phi \cdot,(\operatorname{trace}\langle V, d \phi \cdot\rangle d \phi \cdot)\rangle d \phi \cdot-2|d \phi|^{2} \operatorname{trace}\langle d \phi \cdot, V\rangle d \phi \cdot \\
& +2\langle d V, d \phi\rangle \tau(\phi)-|d \phi|^{2} \Delta^{\phi} V+|d \phi|^{4} V .
\end{aligned}
$$

For this aspect of the theory, the two most important quantities to determine are the nullity and the index, now defined. 


\section{THE INDEX OF BIHARMONIC MAPS IN SPHERES}

Definition 1.3. Let $\phi:(M, g) \rightarrow \mathbb{S}^{n}$ be a biharmonic map. The dimension of the vector space $\left\{V \in C\left(\phi^{-1} T \mathbb{S}^{n}\right): I(V)=0\right\}$ is called the nullity of $\phi$.

The first case to look at, for nullity, is the identity map $1: \mathbb{S}^{n} \rightarrow \mathbb{S}^{n}$, for which the operator $I(V)$ becomes

$$
I(V)=\Delta(\Delta V)-2(n-1) \Delta V+(n-1)^{2} V
$$

and the nullity is the dimension of the vector space $\left\{V \in C\left(T \mathbb{S}^{n}\right): \Delta V=(n-1) V\right\}$. The Hodge decomposition, the eigenvalues of the sphere and the dimension of the space of Killing vector fields imply the following.

Theorem 1.4 [Oni02]. The identity $\mathbf{1}: \mathbb{S}^{n} \rightarrow \mathbb{S}^{n}$ is a biharmonic map of nullity equal to 6 , if $n=2$, and $n(n+1) / 2$, when $n \geqslant 3$.

Definition 1.5. Let $\phi:(M, g) \rightarrow \mathbb{S}^{n}$ be a biharmonic map. The index of $\phi$ is the dimension of the largest of the vector spaces $\left\{V \in C\left(\phi^{-1} T \mathbb{S}^{n}\right):(I(V), V)<0\right\}$. A map of index zero is called stable; otherwise, it is said to be unstable.

Remark 1.6. (i) Any harmonic map is clearly a stable biharmonic map.

(ii) Equation (1) shows that $I$ is a linear elliptic self-adjoint differential operator, and the Hilbert space of sections $L^{2}\left(\phi^{-1} T \mathbb{S}^{n}\right)$ is the orthogonal sum of the finite-dimensional eigenspaces of $I$. Its spectrum consists of real numbers, bounded from below. Therefore, the nullity and the index are finite.

The purpose of this paper is the computation of the index of biharmonic maps constructed in the following manner.

Theorem 1.7. Let $M$ be a compact manifold, $\psi: M \rightarrow \mathbb{S}^{n}(1 / \sqrt{2}) \times\{1 / \sqrt{2}\}$ a non-constant harmonic map and $\mathbf{i}: \mathbb{S}^{n}(1 / \sqrt{2}) \times\{1 / \sqrt{2}\} \rightarrow \mathbb{S}^{n+1}$ the inclusion map. Then $\phi=\mathbf{i} \circ \psi: M \rightarrow \mathbb{S}^{n+1}$ is non-harmonic biharmonic if and only if $e(\psi)=(1 / 2)|d \psi|^{2}$ is constant.

Proof. Let $p \in M$ be an arbitrary point, $\left\{X_{i}\right\}_{i=1, \ldots, m}$ a geodesic frame at $p$, and $\eta$ the unit section of the normal bundle. Using the chain rule for the tension field

$$
\begin{aligned}
\tau(\phi) & =d \mathbf{i}(\tau(\psi))+\operatorname{trace} \nabla d \mathbf{i}(d \psi \cdot, d \psi \cdot) \\
& =-\sum_{i=1}^{m}\left\langle d \psi\left(X_{i}\right), d \psi\left(X_{i}\right)\right\rangle \eta \\
& =-2 e(\psi) \eta,
\end{aligned}
$$

so $\phi$ is not harmonic. Here the second fundamental form of the inclusion is $\nabla d \mathbf{i}(X, Y)=B(X, Y)=$ $-\langle X, Y\rangle \eta$.

By a straightforward computation, we obtain, at the point $p$, that

$$
\frac{1}{2} \nabla_{X_{i}}^{\phi} \nabla_{X_{i}}^{\phi} \tau(\phi)=-\left(X_{i} X_{i} e(\psi)\right) \eta+e(\psi)\left\langle d \psi\left(X_{i}\right), d \psi\left(X_{i}\right)\right\rangle \eta-2 d \phi\left[\left(X_{i} e(\psi)\right) X_{i}\right]-e(\psi) \nabla d \psi\left(X_{i}, X_{i}\right)
$$

and

$$
-R^{\mathbb{S}^{n+1}}\left(d \phi\left(X_{i}\right), \tau(\phi)\right) d \phi\left(X_{i}\right)=\left\langle d \psi\left(X_{i}\right), d \psi\left(X_{i}\right)\right\rangle \tau(\phi)
$$

Thus

$$
\frac{1}{2} \tau^{2}(\phi)=(\Delta e(\psi)) \eta-2 d \phi(\operatorname{grad} e(\psi)) .
$$

Now, the map $\phi$ is biharmonic if and only if

$$
\Delta e(\psi)=0 \quad \text { and } \quad d \phi(\operatorname{grad} e(\psi))=0,
$$

and the theorem follows from the compactness of $M$. 


\section{E. Loubeau And C. Oniciuc}

Remark 1.8. For the sake of clarity, we will drop the reference to the height $\{1 / \sqrt{2}\}$ and denote by $\mathbb{S}^{n}(1 / \sqrt{2})$ the sphere $\mathbb{S}^{n}(1 / \sqrt{2}) \times\{1 / \sqrt{2}\}$.

In the general case we choose to study, we consider a minimal (i.e. harmonic) isometric immersion of a compact Riemannian manifold into the unit sphere and adapt its radius to obtain $\psi:\left(M^{m}, g\right) \rightarrow$ $\mathbb{S}^{n}(1 / \sqrt{2})$, of constant energy density $m / 2$. This is then composed with the inclusion of $\mathbb{S}^{n}(1 / \sqrt{2})$ into $\mathbb{S}^{n+1}$, in order to attain, according to Theorem 1.7, a non-harmonic biharmonic map $\phi$ : $\left(M^{m}, g\right) \rightarrow \mathbb{S}^{n+1}$, the stability of which we will analyse. This framework will remain throughout the paper.

Our general modus operandi will be to look at the pull-back bundle $\phi^{-1} T \mathbb{S}^{n+1}$ in terms of three sub-bundles:

(i) the one-dimensional normal sub-bundle, spanned by the vector field $\eta$ tangent to $\mathbb{S}^{n+1}$ but normal to the sphere $\mathbb{S}^{n}(1 / \sqrt{2})$;

(ii) the image of the tangent space of the domain, i.e. $d \phi(T M)$, named the tangent sub-bundle;

(iii) the subspace consisting of vector fields tangent to $\mathbb{S}^{n}(1 / \sqrt{2})$ but orthogonal to the image of the map and referred to as the vertical sub-bundle.

\section{The normal sub-bundle}

As the image of $\phi$ actually lies in $\mathbb{S}^{n}(1 / \sqrt{2})$, we start by examining vector fields of $C\left(\phi^{-1} T \mathbb{S}^{n+1}\right)$ of the form $V=f \eta, f$ being a function on $M$ and $\eta(p)=(\psi(p),-1 / \sqrt{2}), \forall p \in M$, i.e. $V$ is normal to the hypersphere $\mathbb{S}^{n}(1 / \sqrt{2})$.

Proposition 2.1. Take $V=f \eta, f \in C^{\infty}(M)$. Then

$$
(I(V), V)=\int_{M}\left(\left|\Delta^{\phi} V-m V\right|^{2}-4 m^{2}|V|^{2}\right) v_{g} .
$$

Furthermore, if $\Delta f=\lambda f$, then

$$
(I(V), V)=\left(\lambda^{2}+4 \lambda-4 m^{2}\right) \int_{M} f^{2} v_{g}
$$

Proof. When $V=f \eta$, the various elements of (1) become

$$
\begin{gathered}
\tau(\phi)=-m \eta, \quad \operatorname{trace}\langle V, d \phi \cdot\rangle d \phi \cdot=0, \quad|d \phi|^{2}=m, \\
\langle d \tau(\phi), d \phi\rangle=-m^{2}, \quad|\tau(\phi)|^{2}=m^{2}, \quad \operatorname{trace}\langle V, d \tau(\phi) \cdot\rangle d \phi \cdot=0, \\
\operatorname{trace}\langle\tau(\phi), d V \cdot\rangle d \phi \cdot=-m d \phi(\operatorname{grad} f), \quad\langle\tau(\phi), V\rangle \tau(\phi)=m^{2} V, \\
\operatorname{trace}\left\langle d \phi \cdot, \Delta^{\phi} V\right\rangle d \phi \cdot=\left(\Delta^{\phi} V\right)^{\mathrm{T}}, \quad\langle d V, d \phi\rangle \tau(\phi)=-m^{2} V,
\end{gathered}
$$

and

$$
I(V)=\Delta^{\phi}\left(\Delta^{\phi} V\right)-2 m \Delta^{\phi} V-3 m^{2} V+2 m d \phi(\operatorname{grad} f)+\left(\Delta^{\phi} V\right)^{\mathrm{T}} .
$$

As $V$ is normal to $\mathbb{S}^{n}(1 / \sqrt{2})$,

$$
\langle I(V), V\rangle=\left\langle\Delta^{\phi}\left(\Delta^{\phi} V\right), V\right\rangle-2 m\left\langle\Delta^{\phi} V, V\right\rangle-3 m^{2}|V|^{2},
$$

and integrating by parts yields

$$
(I(V), V)=\int_{M}\left(\left|\Delta^{\phi} V-m V\right|^{2}-4 m^{2}|V|^{2}\right) v_{g} .
$$

To conclude, we observe that

$$
\Delta^{\phi} V=\Delta^{\phi}(f \eta)=(\Delta f) \eta-2 d \phi(\operatorname{grad} f)+m f \eta .
$$




\section{THE INDEX OF BIHARMONIC MAPS IN SPHERES}

Remark 2.2. A crucial observation, for this section, is that if $V=f \eta$ and $W=g \eta$, where $f$ and $g$ are orthogonal eigenfunctions, then $(I(V), W)=0$. So counting the eigenfunctions for which $(I(f \eta), f \eta)$ is negative, together with their multiplicities, one computes the index on the normal sub-bundle.

The principal significance of Proposition 2.1 is to reveal the influence of the small eigenvalues of $\Delta$ on the index of $\phi$.

Corollary 2.3. Let $V=f \eta$ and $\Delta f=\lambda f$. Then $(I(V), V)$ is negative if and only if $\lambda \in$ $\left[0,2\left(\sqrt{m^{2}+1}-1\right)[\right.$.

Example 2.4 (Generalised Veronese map). The well-known Veronese map $\mathbb{S}^{2}(\sqrt{3}) \rightarrow \mathbb{S}^{4}$ is generalised by the immersion of $\mathbb{S}^{m}(\sqrt{2(m+1) / m})$ into $\mathbb{S}^{m+p}$, where $p=(m-1)(m+2) / 2$ (cf. [CdCK70]).

We modify the radius, compose with the inclusion of $\mathbb{S}^{n}(1 / \sqrt{2})$ and obtain a non-harmonic biharmonic map $\phi: \mathbb{S}^{m}(\sqrt{(m+1) / m}) \rightarrow \mathbb{S}^{m+p+1}$. As $\left(\mathbb{S}^{m}(\sqrt{(m+1) / m}), g_{\text {can }}\right)$ is isometric to $\left(\mathbb{S}^{m},[(m+1) / m] g_{\text {can }}\right)$, its eigenvalues are $\left\{\lambda_{k}=[m /(m+1)] k(m+k-1): k \in \mathbb{N}\right\}$, with multiplicity $[(2 k+m-1)(k+m-2) !] /[k !(m-1) !]$ (see [ER93]), and, whatever the dimension, $\lambda_{1}<2\left(\sqrt{m^{2}+1}-1\right)$ while $\lambda_{k}>2\left(\sqrt{m^{2}+1}-1\right), \forall k \geqslant 2$. So, for normal vector fields, contributions to the index of $\phi$ come only from the first two eigenvalues, $\lambda_{0}$ and $\lambda_{1}$, making it greater than or equal to $1+(m+1)=m+2$.

Furthermore, since the generalised Veronese map is quadratic, it also defines a biharmonic map from $\mathbb{R} \mathrm{P}^{m}$, equipped with the metric $[(m+1) / m] g_{\text {can }}$, into $\mathbb{S}^{m+p+1}$. But the spectrum, in this case, is reduced to $\left\{\tilde{\lambda}_{k}=[m /(m+1)] 2 k(m+2 k-1): k \in \mathbb{N}\right\}$ and, as $\tilde{\lambda}_{1}$ is larger than $2\left(\sqrt{m^{2}+1}-1\right)$, only $\eta$ adds to the index.

Example 2.5 (Generalised Clifford torus). An extension to higher dimensions of the Clifford torus is the minimal Riemannian immersion $\mathbb{S}^{l}\left(\frac{1}{2}\right) \times \mathbb{S}^{l}\left(\frac{1}{2}\right) \rightarrow \mathbb{S}^{m+1}(1 / \sqrt{2})$, with $m=2 l$, which gives rise to a non-harmonic biharmonic map $\phi: \mathbb{S}^{l}\left(\frac{1}{2}\right) \times \mathbb{S}^{l}\left(\frac{1}{2}\right) \rightarrow \mathbb{S}^{m+2}$.

The eigenvalues of a product being the sum of the eigenvalues of each factor, the spectrum of the Laplacian on $\mathbb{S}^{l}\left(\frac{1}{2}\right) \times \mathbb{S}^{l}\left(\frac{1}{2}\right)$ is $\left\{\lambda_{k}=4(p(l+p-1)+q(l+q-1)): p, q \in \mathbb{N}, p+q=k\right\}$. So $\lambda_{1}=4 l=2 m$ is greater than $2\left(\sqrt{m^{2}+1}-1\right)$, and, among normal vector fields, only $\eta$ is counted in the index.

Since the spectrum of the Laplacian invariably contains the eigenvalue 0 , we obtain the following theorem.

Theorem 2.6. Let $\left(M^{m}, g\right)$ be a compact Riemannian manifold. A biharmonic map $\phi:\left(M^{m}, g\right) \rightarrow$ $\mathbb{S}^{n+1}$, obtained as the composition of a minimal isometric immersion $\psi:\left(M^{m}, g\right) \rightarrow \mathbb{S}^{n}(1 / \sqrt{2})$ and the inclusion $\mathbb{S}^{n}(1 / \sqrt{2}) \rightarrow \mathbb{S}^{n+1}$, has a strictly positive index, i.e. is unstable.

Remark 2.7. This result is clearly reminiscent of the instability of harmonic maps into the sphere [Leu82].

To further refine Corollary 2.3, one can attempt to control the value of the first non-zero eigenvalue, $\lambda_{1}$, through geometrical constraints. Before anything else, note the upper bound $2 m$ on $\lambda_{1}$, imposed by the hypotheses on $\psi$. Indeed, immersing $\mathbb{S}^{n}(1 / \sqrt{2}) \stackrel{j}{\rightarrow} \mathbb{R}^{n+1}$ makes each component of the map $j \circ \psi: M \rightarrow \mathbb{R}^{n+1}$ an eigenfunction of the Laplacian on $(M, g)$, of eigenvalue $2 m$, hence the bound on $\lambda_{1}$.

Given that many examples are built from spheres, the following helpful condition is inspired by Einstein manifolds.

Proposition 2.8. Let $\psi:(M, g) \rightarrow \mathbb{S}^{n}(1 / \sqrt{2})$ be a minimal Riemannian immersion and $\phi:$ $(M, g) \rightarrow \mathbb{S}^{n+1}$ the biharmonic map constructed above. Assume that $\operatorname{Ricci}^{M} \geqslant \kappa g(\kappa>0)$ and let $\kappa(m)=[2(m-1) / m]\left(\sqrt{m^{2}+1}-1\right)$. If $\kappa \geqslant \kappa(m)$, then the contribution of normal vector fields to the index of $\phi$ is 1 . 


\section{E. Loubeau and C. Oniciuc}

Proof. All hinges on a theorem of Lichnerowicz [Lic58], stating that $\operatorname{Ricci}^{M} \geqslant \kappa g$ implies $\lambda_{1} \geqslant$ $[m /(m-1)] \kappa$. By Corollary 2.3, we only need to ensure that $[m /(m-1)] \kappa \geqslant 2\left(\sqrt{m^{2}+1}-1\right)$.

Example 2.9 (Totally geodesic inclusion). A very simple example of a minimal isometric immersion into the sphere is the totally geodesic inclusion of a lower-dimension sphere in the equator, $\mathbb{S}^{m}(1 / \sqrt{2}) \rightarrow \mathbb{S}^{n}(1 / \sqrt{2})$, with $m \leqslant n$, producing a non-harmonic biharmonic map $\phi: \mathbb{S}^{m}(1 / \sqrt{2}) \rightarrow$ $\mathbb{S}^{n+1}$.

The sphere $\mathbb{S}^{m}(1 / \sqrt{2})$ being isometric to $\left(\mathbb{S}^{m}, \frac{1}{2} g_{\text {can }}\right)$, we can apply the above proposition for $\kappa=2(m-1)$, and deduce that solely the vector field $\eta$ will contribute to the index of the biharmonic map $\phi$ constructed from this totally geodesic inclusion.

Remark 2.10. Although the projective space of Example 2.4 and the product of spheres of Example 2.5 are Einstein manifolds, and therefore admit a constant $\kappa$ satisfying Ricci $\geqslant \kappa g$, in both instances $\kappa<\kappa(m)$.

\section{The tangent sub-bundle}

Vector fields of the tangent sub-bundle are in the image of $T M$ by $d \phi$ and can be written $V=$ $d \phi(X), X \in C(T M)$.

Proposition 3.1. Let $V=d \phi(X)$ be a tangential vector field. Then

$$
(I(V), V)=\int_{M}\left(\left|\Delta^{\phi} V+(1-m) V\right|^{2}-m^{2}|V|^{2}\right) v_{g} .
$$

Proof. As $\phi$ is an isometric immersion and $V$ is in $d \phi(T M)$, and therefore normal to $\eta$ and $\tau(\phi)$, the components of (1) are

$$
\begin{gathered}
\tau(\phi)=-m \eta, \quad \operatorname{trace}\langle V, d \phi \cdot\rangle d \phi \cdot=V, \quad|d \phi|^{2}=m, \\
\langle d \tau(\phi), d \phi\rangle=-m^{2}, \quad|\tau(\phi)|^{2}=m^{2}, \quad \operatorname{trace}\langle V, d \tau(\phi) \cdot\rangle d \phi \cdot=-m V, \\
\operatorname{trace}\langle\tau(\phi), d V \cdot\rangle d \phi \cdot=m V, \quad\langle\tau(\phi), V\rangle \tau(\phi)=0, \quad \operatorname{trace}\left\langle d \phi \cdot, \Delta^{\phi} V\right\rangle d \phi \cdot=\left(\Delta^{\phi} V\right)^{\mathrm{T}}, \\
\operatorname{trace}\langle d \phi \cdot,(\operatorname{trace}\langle V, d \phi \cdot\rangle d \phi \cdot)\rangle d \phi \cdot=V, \quad\langle d V, d \phi\rangle=\operatorname{div} X,
\end{gathered}
$$

and consequently

$$
I(V)=\Delta^{\phi}\left(\Delta^{\phi} V\right)+(1-2 m) \Delta^{\phi} V+\left(\Delta^{\phi} V\right)^{\mathrm{T}}+(1-2 m) V-2 m(\operatorname{div} X) \eta .
$$

Since $V$ is normal to $\eta$ and tangent to the image of $M$, then

$$
\langle I(V), V\rangle=\left\langle\Delta^{\phi}\left(\Delta^{\phi} V\right), V\right\rangle+2(1-m)\left\langle\Delta^{\phi} V, V\right\rangle+(1-2 m)|V|^{2},
$$

and after an integration by parts, the proposition follows.

Exploiting (3) requires a kind of Bochner formula linking the Laplacian of the pull-back bundle to the Hodge-de Rham Laplacian $\Delta_{\mathrm{H}}$ on vector fields tangent to $M$.

Proposition 3.2. Let $X$ be a vector field tangent to $M$ and $V=d \phi(X)$ a vector field of $\phi^{-1} T \mathbb{S}^{n+1}$. Then

$$
\begin{aligned}
(I(V), V)= & \int_{M}\left(4|\operatorname{trace} \nabla d \psi(\nabla . X, \cdot)|^{2}+4(\operatorname{div} X)^{2}\right. \\
& \left.+\left|\Delta_{\mathrm{H}}(X)-2 \operatorname{Ricci}(X)+m X\right|^{2}-m^{2}|X|^{2}\right) v_{g} .
\end{aligned}
$$

Proof. Choosing a geodesic frame $\left\{X_{i}\right\}_{i=1, \ldots, m}$ around $p \in M$ means that, at $p, \nabla_{X_{i}} X_{j}=0, \forall i, j=$ $1, \ldots, m$, and

$$
\Delta^{\phi} V=-\sum_{i=1}^{m} \nabla_{X_{i}}^{\phi} \nabla_{X_{i}}^{\phi} V
$$


Moreover, the second fundamental form of the injection $\mathbf{i}$ of $\mathbb{S}^{n}(1 / \sqrt{2})$ is $B(X, Y)=-\langle X, Y\rangle \eta$, so

$$
\nabla_{X_{i}}^{\phi} V=\nabla_{X_{i}}^{\psi} d \psi(X)-\left\langle X_{i}, X\right\rangle \eta
$$

Composing the preceding expressions implies that

$$
\begin{aligned}
\nabla_{X_{i}}^{\phi} \nabla_{X_{i}}^{\phi} V= & \nabla_{X_{i}}^{\phi} \nabla_{X_{i}}^{\psi} d \psi(X)-\left\langle X_{i}, \nabla_{X_{i}} X\right\rangle \eta-\left\langle X_{i}, X\right\rangle d \phi\left(X_{i}\right) \\
= & \nabla d \mathbf{i}\left(d \psi\left(X_{i}\right), \nabla_{X_{i}}^{\psi} d \psi(X)\right)+\nabla_{X_{i}}^{\psi} \nabla_{X_{i}}^{\psi} d \psi(X) \\
& -\left\langle X_{i}, \nabla_{X_{i}} X\right\rangle \eta-\left\langle X_{i}, X\right\rangle d \phi\left(X_{i}\right) \\
= & \nabla_{X_{i}}^{\psi} \nabla_{X_{i}}^{\psi} d \psi(X)-\left\langle d \psi\left(X_{i}\right), \nabla d \psi\left(X_{i}, X\right)+d \psi\left(\nabla_{X_{i}} X\right)\right\rangle \eta \\
& -\left\langle X_{i}, \nabla_{X_{i}} X\right\rangle \eta-\left\langle X_{i}, X\right\rangle d \phi\left(X_{i}\right) \\
= & \nabla_{X_{i}}^{\psi} \nabla_{X_{i}}^{\psi} d \psi(X)-2\left\langle X_{i}, \nabla_{X_{i}} X\right\rangle \eta-\left\langle X_{i}, X\right\rangle d \phi\left(X_{i}\right) .
\end{aligned}
$$

We conclude that

$$
\Delta^{\phi} V=\Delta^{\psi} V+2(\operatorname{div} X) \eta+V .
$$

The second half of the proof consists of using the harmonicity of $\psi$ and the curvature tensor of $M$ to express $\Delta^{\psi} V$ in terms of $\nabla d \psi$. Thus

$$
\begin{aligned}
\Delta^{\psi} V & =-\sum_{i=1}^{m} \nabla_{X_{i}}^{\psi} \nabla_{X_{i}}^{\psi} V \\
& =-\sum_{i=1}^{m}\left(\nabla_{X_{i}}^{\psi}\left[\nabla d \psi\left(X_{i}, X\right)\right]+\nabla d \psi\left(X_{i}, \nabla_{X_{i}} X\right)+d \psi\left(\nabla_{X_{i}} \nabla_{X_{i}} X\right)\right),
\end{aligned}
$$

because $\nabla_{X_{i}}^{\psi} V=\nabla d \psi\left(X_{i}, X\right)+d \psi\left(\nabla_{X_{i}} X\right)$.

But we have

$$
\begin{aligned}
\nabla_{X_{i}}^{\psi}\left[\nabla d \psi\left(X_{i}, X\right)\right]= & \nabla_{X_{i}}^{\psi}\left[\nabla d \psi\left(X, X_{i}\right)\right] \\
= & \nabla_{X_{i}}^{\psi} \nabla_{X}^{\psi} d \psi\left(X_{i}\right)-\nabla_{X_{i}}^{\psi} d \psi\left(\nabla_{X} X_{i}\right) \\
= & \nabla_{X}^{\psi} \nabla_{X_{i}}^{\psi} d \psi\left(X_{i}\right)+\nabla_{\left[X_{i}, X\right]}^{\psi} d \psi\left(X_{i}\right)+R^{\psi}\left(X_{i}, X\right) d \psi\left(X_{i}\right) \\
& -\nabla d \psi\left(X_{i}, \nabla_{X} X_{i}\right)-d \psi\left(\nabla_{X_{i}} \nabla_{X} X_{i}\right) \\
= & \nabla_{X}^{\psi} \nabla_{X_{i}}^{\psi} d \psi\left(X_{i}\right)+\nabla_{\left[X_{i}, X\right]}^{\psi} d \psi\left(X_{i}\right)+R^{\psi}\left(X_{i}, X\right) d \psi\left(X_{i}\right) \\
& -d \psi\left(\nabla_{X} \nabla_{X_{i}} X_{i}+\nabla_{\left[X_{i}, X\right]} X_{i}+R\left(X_{i}, X\right) X_{i}\right) \\
= & \nabla_{X}^{\psi} \nabla_{X_{i}}^{\psi} d \psi\left(X_{i}\right)+\nabla_{\left[X_{i}, X\right]}^{\psi} d \psi\left(X_{i}\right)+2\left\{\left\langle X_{i}, X\right\rangle d \psi\left(X_{i}\right)-d \psi(X)\right\} \\
& -\nabla_{X}^{\psi} d \psi\left(\nabla_{X_{i}} X_{i}\right)+\nabla d \psi\left(X, \nabla_{X_{i}} X_{i}\right)-d \psi\left(\nabla_{\left[X_{i}, X\right]} X_{i}\right)-d \psi\left(R\left(X_{i}, X\right) X_{i}\right) \\
= & \nabla_{X}^{\psi}\left[\nabla d \psi\left(X_{i}, X_{i}\right)\right]+\nabla d \psi\left(\left[X_{i}, X\right], X_{i}\right) \\
& +2\left(\left\langle X_{i}, X\right\rangle d \psi\left(X_{i}\right)-d \psi(X)\right)-d \psi\left(R\left(X_{i}, X\right) X_{i}\right) .
\end{aligned}
$$

Since $\left[X_{i}, X\right]=\nabla_{X_{i}} X$ at $p$, we obtain

$$
\begin{aligned}
\Delta^{\psi} V & =-2 \sum_{i=1}^{m} \nabla d \psi\left(\nabla_{X_{i}} X, X_{i}\right)+d \psi\left(2(m-1) X-\operatorname{trace} \nabla^{2} X-\operatorname{Ricci}(X)\right) \\
& =-2 \sum_{i=1}^{m} \nabla d \psi\left(\nabla_{X_{i}} X, X_{i}\right)+d \psi\left(2(m-1) X+\Delta_{\mathrm{H}}(X)-2 \operatorname{Ricci}(X)\right) .
\end{aligned}
$$

The proposition then follows from (3), (4) and (5). 


\section{E. Loubeau And C. Oniciuc}

Theorem 3.3. Let $\left(M^{m}, g\right), m \geqslant 2$, be a compact Riemannian manifold and $\phi=\mathbf{i} \circ \psi: M \rightarrow \mathbb{S}^{n+1}$ a biharmonic map, forged as the composition of a minimal isometric immersion $\psi$ and the inclusion $\mathbf{i}$. Let $V$ be a vector field of the pull-back bundle $\phi^{-1} T \mathbb{S}^{n+1}$, such that $V=d \phi(X), X \in C(T M)$. If $\operatorname{dim} M \leqslant 4$ or $\operatorname{div}(X)=0$, then $(I(V), V) \geqslant 0$.

Proof. Let $V=d \psi(X), X \in C(T M)$. Via the Yano formula [Yan52],

we know that

$$
\int_{M}(\operatorname{div} X)^{2}-\left\langle\operatorname{trace} \nabla^{2} X+\operatorname{Ricci}(X), X\right\rangle v_{g}=\int_{M} \frac{1}{2}\left|L_{X} g\right|^{2} v_{g}
$$

$$
(I(V), V) \geqslant \int_{M}\left(\left|\operatorname{trace} \nabla^{2} X+\operatorname{Ricci}(X)\right|^{2}+m\left|L_{X} g\right|^{2}+2(2-m)(\operatorname{div} X)^{2}\right) v_{g} .
$$

Recall that, for $X$ tangent to $(M, g)$,

$$
\left|L_{X} g\right|^{2} \geqslant \frac{4}{m}(\operatorname{div} X)^{2}
$$

since, for a local orthonormal frame field $\left\{X_{i}\right\}_{i=1, \ldots, m}$ on $M$,

$$
\begin{aligned}
\left|L_{X} g\right|^{2} & =\sum_{i, j=1}^{m}\left(\left(L_{X} g\right)\left(X_{i}, X_{j}\right)\right)^{2}=\sum_{i, j=1}^{m}\left(\left\langle\nabla_{X_{i}} X, X_{j}\right\rangle+\left\langle\nabla_{X_{j}} X, X_{i}\right\rangle\right)^{2} \\
& \geqslant 4 \sum_{i=1}^{m}\left(\left\langle\nabla_{X_{i}} X, X_{i}\right\rangle\right)^{2},
\end{aligned}
$$

and by the Cauchy inequality

$$
\left|L_{X} g\right|^{2} \geqslant \frac{4}{m}\left(\sum_{i=1}^{m}\left\langle\nabla_{X_{i}} X, X_{i}\right\rangle\right)^{2}=\frac{4}{m}(\operatorname{div} X)^{2} .
$$

Substituting in (6), we obtain

$$
(I(V), V) \geqslant 2(4-m) \int_{M}(\operatorname{div} X)^{2} v_{g} .
$$

Remark 3.4. Using the fact that, for a Killing vector field $X$,

$$
\sum_{i=1}^{m} \nabla d \psi\left(\nabla_{X_{i}} X, X_{i}\right)=0
$$

and trace $\nabla^{2} X+$ Ricci $X=0$, one can also show that $I(V)=0$. Moreover, the converse also holds if $m \leqslant 4$ or $\operatorname{div} X=0$, since, in these cases, $I(V)=0$ implies $L_{X} g=0$ by (6), i.e. $X$ is a Killing vector field.

Corollary 3.5. The nullity of the biharmonic map $\phi$ is bounded from below by the dimension of $\operatorname{Isom}(M, g)$.

We can complement Theorem 3.3 when the first eigenvalue of the Laplacian is large enough.

Corollary 3.6. Let $\phi: M^{m} \rightarrow \mathbb{S}^{n+1}$ be a biharmonic map, fashioned as before, and $\lambda_{1}$ the smallest non-zero eigenvalue of the Laplacian on $(M, g)$. If $\lambda_{1} \geqslant m^{2} / 4$, then $(I(V), V) \geqslant 0$ for all vector fields $V \in C\left(\phi^{-1} T \mathbb{S}^{n+1}\right)$ such that $V=d \phi(\operatorname{grad} f), \Delta f=\lambda f$.

Proof. Let $V=d \phi(X)$ and $X=\operatorname{grad} f$, where $\Delta f=\lambda_{1} f$. The proposition comes from the inequalities

$$
\begin{aligned}
(I(V), V)= & \int_{M}\left(4|\operatorname{trace} \nabla d \psi(\nabla . X, \cdot)|^{2}+4(\operatorname{div} X)^{2}\right. \\
& \left.+\left|\Delta_{\mathrm{H}}(X)-2 \operatorname{Ricci}(X)+m X\right|^{2}-m^{2}|X|^{2}\right) v_{g}
\end{aligned}
$$


THE INDEX OF BIHARMONIC MAPS IN SPHERES

$$
\begin{aligned}
& \geqslant \int_{M}\left(4(\operatorname{div} X)^{2}-m^{2}|X|^{2}\right) v_{g} \\
& =\int_{M}\left(4(\Delta f)^{2}-m^{2}|\operatorname{grad} f|^{2}\right) v_{g} \\
& =\lambda_{1}\left(4 \lambda_{1}-m^{2}\right) \int_{M} f^{2} v_{g} .
\end{aligned}
$$

Example 3.7 (Generalised Clifford torus). The first eigenvalue of the Laplacian on $\mathbb{S}^{l}\left(\frac{1}{2}\right) \times \mathbb{S}^{l}\left(\frac{1}{2}\right)$ is $4 l$, so, for the biharmonic map $\phi: \mathbb{S}^{l}\left(\frac{1}{2}\right) \times \mathbb{S}^{l}\left(\frac{1}{2}\right) \rightarrow \mathbb{S}^{m+2}(m=2 l),(I(V), V)$ will be positive for vector fields $V=d \phi(\operatorname{grad} f), f$ an eigenfunction of the Laplacian, when $2 m \geqslant m^{2} / 4$, that is $m \leqslant 8$.

Owing to the nature of our construction, we can link the stability of the biharmonic map $\phi$ : $M \rightarrow \mathbb{S}^{n+1}$ to that of the identity.

THEOREM 3.8. Let $\psi$ be a minimal isometric immersion from a compact Riemannian manifold $(M, g)$ into $\mathbb{S}^{n}(1 / \sqrt{2}), \phi: M \rightarrow \mathbb{S}^{n+1}$ the associated biharmonic map and $I$ its second variation operator. If the identity $\mathbf{1}:(M, g) \rightarrow(M, g)$ is stable, as a harmonic map, then $(I(V), V)$ is positive for any vector field $V$ of $\phi^{-1} T \mathbb{S}^{n+1}$ tangent to $\phi(M)$.

Proof. Writing a vector field $V \in C\left(\phi^{-1} T \mathbb{S}^{n+1}\right)$ as $d \phi(X), X \in C(T M)$, yields

$$
(I(V), V)=\int_{M}\left(\left|\Delta^{\phi} V+(1-m) V\right|^{2}-m^{2}|V|^{2}\right) v_{g}
$$

with $\Delta^{\phi} V=\Delta^{\psi} V+2(\operatorname{div} X) \eta+V$. On the other hand, the Jacobi operator of $\psi$ is

$$
J^{\psi}(V)=\Delta^{\psi} V+\operatorname{trace} R^{\mathbb{S}^{n}(1 / \sqrt{2})}(d \psi, V) d \psi,
$$

with

$$
\begin{aligned}
\operatorname{trace} R^{\mathbb{S}^{n}(1 / \sqrt{2})}(d \psi, V) d \psi & =\sum_{i=1}^{m} R^{\mathbb{S}^{n}(1 / \sqrt{2})}\left(d \psi\left(X_{i}\right), V\right) d \psi\left(X_{i}\right) \\
& =2 \sum_{i=1}^{m}\left(\left\langle X_{i}, X\right\rangle d \psi\left(X_{i}\right)-\left\langle X_{i}, X_{i}\right\rangle V\right) \\
& =2(1-m) V,
\end{aligned}
$$

so that

$$
J^{\psi}(V)=\Delta^{\psi} V+2(1-m) V .
$$

Therefore we obtain

$$
\Delta^{\phi} V=J^{\psi}(V)+2(\operatorname{div} X) \eta+(2 m-1) V
$$

and

$$
\Delta^{\phi} V+(1-m) V=J^{\psi}(V)+2(\operatorname{div} X) \eta+m V .
$$

Integrating shows that

$$
\begin{aligned}
(I(V), V) & =\int_{M}\left(\left|J^{\psi}(V)+m V\right|^{2}+4(\operatorname{div} X)^{2}-m^{2}|V|^{2}\right) v_{g} \\
& =\int_{M}\left(\left|J^{\psi}(V)\right|^{2}+4(\operatorname{div} X)^{2}+2 m\left\langle J^{\psi}(V), V\right\rangle\right) v_{g} \\
& \geqslant 2 m \int_{M}\left\langle J^{\psi}(V), V\right\rangle v_{g}=2 m \int_{M}\left\langle J^{\mathbf{1}}(X), X\right\rangle v_{g} .
\end{aligned}
$$

Remark 3.9. The stability of the identity of $(M, g)$ is, in fact, equivalent to $\left(J^{\psi}(V), V\right)$ positive for any section $V$ of $\psi^{-1} T \mathbb{S}^{n}(1 / \sqrt{2})$ and tangent to $\phi(M)$. In this respect, $\phi$ inherits part of the stability of $\psi$. 


\section{E. Loubeau and C. Oniciuc}

Example 3.10 (Generalised Clifford torus, revisited). The manifold $\mathbb{S}^{l}\left(\frac{1}{2}\right) \times \mathbb{S}^{l}\left(\frac{1}{2}\right)$ being Einstein of constant $\kappa=4(l-1)$, its identity map will be stable if and only if $2 \kappa \leqslant \lambda_{1}$ (see [Smi75]), i.e. provided $l=1$ or 2 . For these values of $l$, the index of the biharmonic map $\phi: \mathbb{S}^{l}\left(\frac{1}{2}\right) \times \mathbb{S}^{l}\left(\frac{1}{2}\right) \rightarrow \mathbb{S}^{2 l+2}$, as we already knew, has no contribution from vector fields of the tangent sub-bundle.

When $l>2$, we need a finer analysis of the sign of $(I(V), V)$. Staying with a section $V$ of $\phi^{-1} T \mathbb{S}^{2 l+2}$, such that $V=d \phi(X), X=\operatorname{grad} f$ with $\Delta f=\lambda f$, we know that

$$
\begin{aligned}
(I(V), V) & \geqslant \int_{\mathbb{S}^{l}(1 / 2) \times \mathbb{S}^{l}(1 / 2)}\left(4(\operatorname{div} X)^{2}+\left|\Delta_{\mathrm{H}}(X)-2 \operatorname{Ricci}(X)+m X\right|^{2}-m^{2}|X|^{2}\right) v_{g} \\
& =\int_{\mathbb{S}^{l}(1 / 2) \times \mathbb{S}^{l}(1 / 2)}\left(4 \lambda^{2} f^{2}+(\lambda-8(l-1)+m)^{2} \lambda f^{2}-m^{2} \lambda f^{2}\right) v_{g} \\
& =\lambda\left(\lambda^{2}+2 \lambda(10-3 m)+8 m^{2}-48 m+64\right) \int_{\mathbb{S}^{l}(1 / 2) \times \mathbb{S}^{l}(1 / 2)} f^{2} v_{g},
\end{aligned}
$$

where $m=2 l$. Therefore, when $P(\lambda)=\lambda^{2}+2 \lambda(10-3 m)+8 m^{2}-48 m+64$ is positive, so is $(I(V), V)$, and, as the first non-zero eigenvalue $\lambda_{1}$ of the Laplace-Beltrami operator on $\mathbb{S}^{l}\left(\frac{1}{2}\right) \times \mathbb{S}^{l}\left(\frac{1}{2}\right)$ is $4 l=2 m$, $P\left(\lambda_{1}\right) \geqslant 0$ if and only if $m \leqslant 8$. The polynomial $P$ is increasing on the interval $[2 m,+\infty$ [ when $m \leqslant 10$, so $P\left(\lambda_{k}\right) \geqslant P\left(\lambda_{1}\right) \geqslant 0, \forall k \geqslant 1$, as long as $m \leqslant 8$ (this reproduces Example 3.7).

For dimensions above $8, P\left(\lambda_{1}\right)$ is negative but $P\left(\lambda_{2}\right)$, and therefore $P\left(\lambda_{k}\right)$ for $k \geqslant 2$, is positive, since $\lambda_{k}=4 p(l+p-1)+4 q(l+q-1)$ where $p+q=k$.

Nonetheless, for $\lambda_{1}$, we can push this investigation a little further, by explicitly computing the term from Proposition 3.2 that was overlooked in inequality (7), i.e. $4 \mid$ trace $\left.\nabla d \psi(\nabla . X, \cdot)\right|^{2}$.

Let $f \in C^{\infty}\left(\mathbb{S}^{l}\left(\frac{1}{2}\right)\right)$ such that $\Delta^{\mathbb{S}^{l}(1 / 2)} f=2 m f$ and $\tilde{f}=f \circ p, p$ being the first projection $p$ :

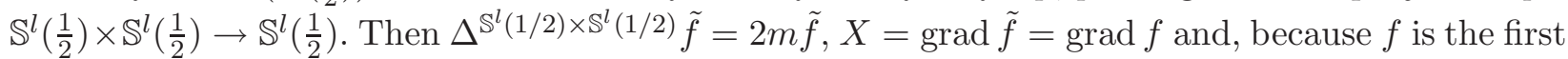
eigenfunction of the Laplacian on a sphere, $\nabla_{X_{a}} X=\nabla_{X_{a}}^{\mathbb{S}^{l}(1 / 2)} \operatorname{grad} f=-4 f X_{a}$, where $\left\{X_{a}\right\}_{a=1, \ldots, l}$ is an orthonormal frame of $\mathbb{S}^{l}\left(\frac{1}{2}\right)$. Then, if $\left\{X_{a}, X_{b}\right\}_{a, b=1, \ldots, l}=\left\{X_{i}\right\}_{i=1, \ldots, 2 l}$ is an orthonormal frame of $\mathbb{S}^{l}\left(\frac{1}{2}\right) \times \mathbb{S}^{l}\left(\frac{1}{2}\right)$

$$
\begin{aligned}
4|\operatorname{trace} \nabla d \psi(\nabla . X, \cdot)|^{2} & =4\left|\sum_{i=1}^{2 l} \nabla d \psi\left(\nabla_{X_{i}} X, X_{i}\right)\right|^{2} \\
& =4\left|\sum_{a=1}^{l} \nabla d \psi\left(\nabla_{X_{a}} X, X_{a}\right)+\sum_{b=1}^{l} \nabla d \psi\left(\nabla_{X_{b}} X, X_{b}\right)\right|^{2} \\
& =4\left|-4 f \sum_{a=1}^{l} \nabla d \psi\left(X_{a}, X_{a}\right)+\sum_{b=1}^{l} \nabla d \psi\left(0, X_{b}\right)\right|^{2} \\
& =64 f^{2}\left|\sum_{a=1}^{l} \sqrt{2} \xi\right|^{2} \\
& =32 m^{2} f^{2}
\end{aligned}
$$

and

$$
(I(V), V)=16 m(m+8) \int_{\mathbb{S}^{l}(1 / 2) \times \mathbb{S}^{l}(1 / 2)} f^{2} v_{g}>0 .
$$

A similar computation yields the same result for $\tilde{f}=f \circ q$ and $\tilde{f}=f \circ p+f \circ q$, where $q$ is the projection on the second factor.

In conclusion, if $V=d \phi(\operatorname{grad} f)$ with $\Delta f=\lambda f$, then $(I(V), V)>0$, whatever the dimension. 


\section{THE INDEX OF BIHARMONIC MAPS IN SPHERES}

Remark 3.11. When our examples have dimension less than 4, calling on Theorem 3.3 is a far easier option.

As in the previous section, when the domain is an Einstein manifold, conditions can be imposed on the Einstein constant to ensure the positivity of $(I(V), V)$.

Proposition 3.12. Let $\left(M^{m}, g\right)$ be an Einstein manifold of constant $\kappa \in \mathbb{R}$, i.e. Ricci $=\kappa g$, and $\phi: M \rightarrow \mathbb{S}^{n+1}$ a biharmonic map constructed as previously. If $\kappa \geqslant(m+2)^{2} / 8$ then $(I(V), V) \geqslant 0$, for $V=d \phi(\operatorname{grad} f), \Delta f=\lambda f$.

Proof. Let $X \in C(T M)$ and $V=d \phi(X)$. Assume that $X=\operatorname{grad} f, f \in C^{\infty}(M)$ and $\Delta f=\lambda f$. By Proposition 3.2

$$
(I(V), V) \geqslant \int_{M}\left(4(\operatorname{div} X)^{2}+\left|\Delta_{\mathrm{H}}(X)-2 \operatorname{Ricci}(X)+m X\right|^{2}-m^{2}|X|^{2}\right) v_{g},
$$

but $\Delta_{\mathrm{H}} X=\Delta_{\mathrm{H}}(\operatorname{grad} f)=\operatorname{grad} \Delta f=\lambda X$, Ricci $X=\kappa X$ and

$$
\begin{aligned}
\int_{M}|X|^{2} v_{g} & =\int_{M}|\operatorname{grad} f|^{2} v_{g}=\int_{M} \lambda f^{2} v_{g}, \\
\int_{M}(\operatorname{div} X)^{2} v_{g} & =\int_{M}(\Delta f)^{2} v_{g}=\lambda^{2} \int_{M} f^{2} v_{g} .
\end{aligned}
$$

Therefore

$$
(I(V), V) \geqslant \lambda\left(\lambda^{2}+2(m+2-2 \kappa) \lambda+4 \kappa^{2}-4 \kappa m\right) \int_{M} f^{2} v_{g}
$$

and, if $\kappa \geqslant(m+2)^{2} / 8, \lambda^{2}+2(m+2-2 \kappa) \lambda+4 \kappa^{2}-4 \kappa m \geqslant 0$, so $(I(V), V)$ is positive.

Example 3.13 (Totally geodesic inclusion). Seeing that the sphere $\mathbb{S}^{m}(1 / \sqrt{2})$ has $\kappa=2(m-1)$ for Einstein constant, we can apply the above proposition to show that the biharmonic map $\phi$ : $\mathbb{S}^{m}(1 / \sqrt{2}) \rightarrow \mathbb{S}^{n+1}$ is stable in the direction of vector fields $d \phi(\operatorname{grad} f)(\Delta f=\lambda f)$, as long as $2(m-1) \geqslant(m+2)^{2} / 8$, i.e. $m \in[2,9]$.

If $m=1$, then $\kappa=0$ and $(I(V), V)$ is always positive for $V=d \phi(\operatorname{grad} f)$.

When $m \geqslant 10$ and $V=d \phi(\operatorname{grad} f)(\Delta f=\lambda f)$,

$$
\begin{aligned}
(I(V), V) & =\int_{\mathbb{S}^{m}(1 / \sqrt{2})}\left(4(\operatorname{div} X)^{2}+\left|\Delta_{\mathrm{H}}(X)-2 \kappa X\right|^{2}+2 m\left\langle\Delta_{\mathrm{H}}(X)-2 \kappa X, X\right\rangle\right) v_{g} \\
& =\lambda\left(\lambda^{2}+6(2-m) \lambda+8(m-1)(m-2)\right) \int_{\mathbb{S}^{m}(1 / \sqrt{2})} f^{2} v_{g},
\end{aligned}
$$

which is always positive as the spectrum of the Laplacian on $\mathbb{S}^{m}(1 / \sqrt{2})$ is $\left\{\lambda_{k}=2 k(m+k-1)\right.$ : $k \in \mathbb{N}\}$.

Example 3.14 (Generalised Veronese map). To the minimal Riemannian immersion of $\mathbb{S}^{m}(\sqrt{2(m+1) / m})$ into $\mathbb{S}^{m+p}, p=(m-1)(m+2) / 2$, Theorem 1.7 associates a non-harmonic biharmonic map $\phi: \mathbb{S}^{m}(\sqrt{(m+1) / m}) \rightarrow \mathbb{S}^{m+p+1}$.

Since $\mathbb{S}^{m}(\sqrt{(m+1) / m})$, equipped as usual with the canonical metric, is an Einstein manifold of constant $\kappa=m(m-1) /(m+1)$, we deduce that if $V=d \phi(X)$ for $X=\operatorname{grad} f, f \in$ $C^{\infty}\left(\mathbb{S}^{m}(\sqrt{(m+1) / m})\right)$ with $\Delta f=\lambda f$, then as we saw in the proof of Proposition 3.12, $(I(V), V)$ is positive as long as $P(\lambda)=\lambda^{2}+2 \lambda(m+2-2 \kappa)+4 \kappa^{2}-4 m \kappa$ is positive.

Recall that the eigenvalues of the Laplacian on $\mathbb{S}^{m}(\sqrt{(m+1) / m})$ are $\left\{\lambda_{k}=[m /(m+1)] k(m+\right.$ $k-1): k \in \mathbb{N}\}$. The roots of $P$ are

$x_{1}=\frac{m^{2}-5 m-2}{m+1}-\sqrt{\frac{m^{3}-3 m^{2}+16 m+4}{m+1}}$ and $x_{2}=\frac{m^{2}-5 m-2}{m+1}+\sqrt{\frac{m^{3}-3 m^{2}+16 m+4}{m+1}}$,

and $\lambda_{k} \geqslant x_{2}, \forall m \geqslant 2$ and $\forall k \geqslant 2$, while $\lambda_{1} \geqslant x_{2}$ only if $m=2,3$ or 4 (as predicted by Theorem 3.3). 


\section{E. Loubeau and C. Oniciuc}

If $m \geqslant 5$, then $\left.\lambda_{1} \in\right] x_{1}, x_{2}$ [ and we need to study in detail the term $\mid$ trace $\left.\nabla d \psi(\nabla . X, \cdot)\right|^{2}$, for $X=\operatorname{grad} f$ with $\Delta f=\lambda_{1} f$. This is, again, made possible by the fact that, on a sphere, the first eigenfunction of the Laplacian takes the form $f(x)=\langle u, x\rangle$ with $u \in \mathbb{R}^{m+1}$, and satisfies $\nabla_{X} \operatorname{grad} f=-[m /(m+1)] f X$. So, for an orthonormal frame field $\left\{X_{i}\right\}_{i=1, \ldots, m}$,

$$
\sum_{i=1}^{m} \nabla d \psi\left(\nabla_{X_{i}} X, X_{i}\right)=-\frac{m}{m+1} f \sum_{i=1}^{m} \nabla d \psi\left(X_{i}, X_{i}\right)=0,
$$

by the harmonicity of $\psi$.

In conclusion, for the biharmonic map obtained from the generalised Veronese map, $(I(V), V)$ is positive when $V=d \phi(\operatorname{grad} f)$ and $\Delta f=\lambda f$, except for $\lambda=\lambda_{1}$.

\section{The vertical sub-bundle}

The third case of vector fields are sections of the pull-back bundle $\phi^{-1} T \mathbb{S}^{n+1}$, tangent to the sphere $\mathbb{S}^{n}(1 / \sqrt{2})$ but orthogonal to the image of the map. For such a vector field $V$, the second variation operator is easily worked out as follows.

Proposition 4.1. Let $V \in C\left(\phi^{-1} T \mathbb{S}^{n+1}\right)$ be orthogonal to $\eta$ and $d \phi(T M)$. Then

$$
(I(V), V)=\int_{M}\left|\Delta^{\phi} V\right|^{2}-2 m\left\langle\Delta^{\phi} V, V\right\rangle v_{g}
$$

Proof. The basic properties of $V$, i.e. $\langle V, \eta\rangle=\langle V, d \phi(X)\rangle=0, \forall X \in T M$, imply that the nonvanishing constituents of (1) are

$$
\begin{aligned}
I(V)= & \Delta^{\phi} \Delta^{\phi} V-\Delta^{\phi}\left(|d \phi|^{2} V\right)+2\langle d \tau(\phi), d \phi\rangle V+|\tau(\phi)|^{2} V-2 \operatorname{trace}\langle V, d \tau(\phi) \cdot\rangle d \phi \cdot \\
& -2 \operatorname{trace}\langle\tau(\phi), d V \cdot\rangle d \phi \cdot+\operatorname{trace}\left\langle d \phi \cdot, \Delta^{\phi} V\right\rangle d \phi \cdot+2\langle d V, d \phi\rangle \tau(\phi)-|d \phi|^{2} \Delta^{\phi} V+|d \phi|^{4} V .
\end{aligned}
$$

Taking the inner product with $V$ produces

$$
\begin{aligned}
\langle I(V), V\rangle & =\left\langle\Delta^{\phi} \Delta^{\phi} V-m \Delta^{\phi} V-2 m^{2} V+m^{2} V-m \Delta^{\phi} V+m^{2} V, V\right\rangle \\
& =\left\langle\Delta^{\phi} \Delta^{\phi} V-2 m \Delta^{\phi} V, V\right\rangle,
\end{aligned}
$$

and integrating by parts yields

$$
(I(V), V)=\int_{M}\left|\Delta^{\phi} V\right|^{2}-2 m\left\langle\Delta^{\phi} V, V\right\rangle v_{g}
$$

Though (8) is generally unworkable, assuming that $m=n-1$ and that the normal bundle of $M$ in $\psi^{-1} T \mathbb{S}^{n}(1 / \sqrt{2})$, i.e. the vertical sub-bundle, is parallelizable enables $\Delta^{\phi} V$ to be expressed as a function of the shape operator.

Proposition 4.2. Let $\psi: M^{m} \rightarrow \mathbb{S}^{n}(1 / \sqrt{2})$ be a minimal isometric immersion and $\phi: M^{m} \rightarrow \mathbb{S}^{n+1}$ the coupled biharmonic map. Assume that $m=n-1$ and that there exists a unit section $\xi$ of the normal bundle of $M$ in $\psi^{-1} T \mathbb{S}^{n}(1 / \sqrt{2})$. Let $V=f \xi, f \in C^{\infty}(M)$ and denote by $A_{\xi}$ the shape operator of $\xi$. Then $\Delta^{\phi} V=\Delta^{\psi} V$ and

$$
\begin{aligned}
\left\langle\Delta^{\psi} V, V\right\rangle & =(\Delta f) f+f^{2}|\nabla d \psi|^{2}, \\
\left|\Delta^{\psi} V\right|^{2} & =\left(\Delta f+|\nabla d \psi|^{2} f\right)^{2}+\left|2 A_{\xi}(d \psi(\operatorname{grad} f))+f \operatorname{trace}\left(\nabla A_{\xi}\right)(d \psi(\cdot), d \psi(\cdot))\right|^{2} .
\end{aligned}
$$

Proof. Let $V=f \xi$ with $f \in C^{\infty}(M)$, so that $\langle V, \eta\rangle=\langle V, d \phi(X)\rangle=0, \forall X \in C(T M)$. The first thing to observe is that, for $X \in C(T M)$,

$$
\begin{aligned}
\nabla_{X}^{\phi} V & =\nabla_{d \phi(X)}^{\mathbb{S}^{n+1}} V=\nabla_{d \psi(X)}^{\mathbb{S}^{n+1}} V=\nabla_{d \psi(X)}^{\mathbb{S}^{n}(1 / \sqrt{2})} V-\langle d \phi(X), V\rangle \eta \\
& =\nabla_{d \psi(X)}^{\mathbb{S}^{n}(1 / \sqrt{2})} V=\nabla_{X}^{\psi} V .
\end{aligned}
$$


Furthermore

$$
\begin{aligned}
\nabla_{Y}^{\phi} \nabla_{X}^{\phi} V & =\nabla_{Y}^{\phi} \nabla_{X}^{\psi} V \\
& =\nabla_{Y}^{\psi} \nabla_{X}^{\psi} V-\left\langle d \psi(Y), \nabla_{X}^{\psi} V\right\rangle \eta \\
& =\nabla_{Y}^{\psi} \nabla_{X}^{\psi} V+\left\langle\nabla_{X}^{\psi} d \psi(Y), V\right\rangle \eta \\
& =\nabla_{Y}^{\psi} \nabla_{X}^{\psi} V+\left\langle\nabla d \psi(X, Y)+d \psi\left(\nabla_{X} Y\right), V\right\rangle \eta \\
& =\nabla_{Y}^{\psi} \nabla_{X}^{\psi} V+\langle\nabla d \psi(X, Y), V\rangle \eta
\end{aligned}
$$

and $\Delta^{\phi} V=\Delta^{\psi} V$, as $\psi$ is harmonic. Now

$$
\begin{aligned}
\nabla_{X}^{\psi}(f \xi) & =(X f) \xi+f \nabla_{X}^{\psi} \xi \\
& =(X f) \xi+f\left(\nabla_{d \psi(X)}^{\perp} \xi-A_{\xi}(d \psi(X))\right) .
\end{aligned}
$$

The second order of differentiation is

$$
\begin{aligned}
\nabla_{Y}^{\psi} \nabla_{X}^{\psi}(f \xi)= & \nabla_{Y}^{\psi}\left((X f) \xi+f\left(\nabla_{d \psi(X)}^{\perp} \xi-A_{\xi}(d \psi(X))\right)\right) \\
= & Y(X f) \xi+(X f)\left(\nabla_{d \psi(Y)}^{\perp} \xi-A_{\xi}(d \psi(Y))\right)+\nabla_{Y}^{\psi}\left(f\left(\nabla_{d \psi(X)}^{\perp} \xi-A_{\xi}(d \psi(X))\right)\right) \\
= & Y(X f) \xi+(X f) \nabla_{d \psi(Y)}^{\perp} \xi-(X f) A_{\xi}(d \psi(Y))+(Y f)\left(\nabla_{d \psi(X)}^{\perp} \xi-A_{\xi}(d \psi(X))\right) \\
& +f\left(\nabla_{d \psi(Y)}^{\perp} \nabla_{d \psi(X)}^{\perp} \xi-A_{\nabla_{\frac{1}{d \psi(X)}}}(d \psi(Y))-\nabla_{d \psi(Y)}\left(A_{\xi}(d \psi(X))\right)\right. \\
& \left.-B\left(d \psi(Y), A_{\xi}(d \psi(X))\right)\right)
\end{aligned}
$$

and

$$
\begin{aligned}
\Delta^{\psi} V= & (\Delta f) \xi-2 \nabla_{d \psi(\operatorname{grad} f)}^{\perp} \xi+2 A_{\xi}(d \psi(\operatorname{grad} f)) \\
& +f\left(\Delta^{\perp} \xi+|\nabla d \psi|^{2} \xi+\sum_{i=1}^{m}\left(A_{\nabla_{d \psi\left(X_{i}\right.}^{\perp}} \xi\right.\right. \\
= & (\Delta f) \xi+2 A_{\xi}(d \psi(\operatorname{grad} f))+f|\nabla d \psi|^{2} \xi+f \sum_{i=1}^{m} \nabla_{d \psi\left(X_{i}\right)}\left(A_{\xi}\left(d \psi\left(X_{i}\right)\right)\right)
\end{aligned}
$$

since $\nabla^{\perp} \xi=0$. As a result

$$
\left\langle\Delta^{\psi} V, V\right\rangle=(\Delta f) f+f^{2}|\nabla d \psi|^{2}
$$

and

$$
\left|\Delta^{\psi} V\right|^{2}=\left(\Delta f+|\nabla d \psi|^{2} f\right)^{2}+\left|2 A_{\xi}(d \psi(\operatorname{grad} f))+f \sum_{i=1}^{m} \nabla_{d \psi\left(X_{i}\right)} A_{\xi}\left(d \psi\left(X_{i}\right)\right)\right|^{2}
$$

Example 4.3 (Generalised Clifford torus). The minimal isometric immersion of the generalised Clifford torus $\mathbb{S}^{l}\left(\frac{1}{2}\right) \times \mathbb{S}^{l}\left(\frac{1}{2}\right) \rightarrow \mathbb{S}^{2 l+1}(1 / \sqrt{2})$ has codimension one and a parallelizable normal bundle, the vector field $\xi$ being defined by $\xi\left(p, p^{\prime}\right)=\sqrt{2}\left(p,-p^{\prime}\right)$ for $\left(p, p^{\prime}\right) \in \mathbb{S}^{l}\left(\frac{1}{2}\right) \times \mathbb{S}^{l}\left(\frac{1}{2}\right)$. One can readily verify that $|\xi|=1$ and $\langle\xi, X\rangle=0, \forall X \in T \mathbb{S}^{l} \times T \mathbb{S}^{l}$.

Furthermore, the shape operator at $\xi$ is

$$
A_{\xi}=\sqrt{2}\left(\begin{array}{cc}
-\mathrm{Id} & 0 \\
0 & \mathrm{Id}
\end{array}\right)
$$




\section{E. Loubeau and C. Oniciuc}

so, for a function $f$ on $\mathbb{S}^{l}\left(\frac{1}{2}\right) \times \mathbb{S}^{l}\left(\frac{1}{2}\right)$ and an orthonormal frame $\left\{X_{i}\right\}_{i=1, \ldots, 2 l}$,

$$
\begin{aligned}
A_{\xi}(d \psi(\operatorname{grad} f)) & =\sum_{i=1}^{2 l} A_{\xi}\left(d \psi\left(X_{i}(f) X_{i}\right)\right) \\
& =-\sqrt{2} \sum_{i=1}^{l} d \psi\left(X_{i}(f) X_{i}\right)+\sqrt{2} \sum_{i=l+1}^{2 l} d \psi\left(X_{i}(f) X_{i}\right)
\end{aligned}
$$

and

$$
\left|A_{\xi}(d \psi(\operatorname{grad} f))\right|^{2}=2|\operatorname{grad} f|^{2} .
$$

Moreover

$$
\begin{aligned}
\operatorname{trace}\left(\nabla A_{\xi}\right)(d \psi(\cdot), d \psi(\cdot)) & =\sum_{i=1}^{2 l}\left(\nabla A_{\xi}\right)\left(d \psi\left(X_{i}\right), d \psi\left(X_{i}\right)\right) \\
& =\sum_{i=1}^{2 l}\left(\nabla_{d \psi\left(X_{i}\right)} A_{\xi}\left(d \psi\left(X_{i}\right)\right)-A_{\xi}\left(\nabla_{d \psi\left(X_{i}\right)} d \psi\left(X_{i}\right)\right)\right) \\
& =0,
\end{aligned}
$$

since $A_{\xi}$ acts as the identity (up to a constant) on each of the factors of $\mathbb{S}^{l}\left(\frac{1}{2}\right) \times \mathbb{S}^{l}\left(\frac{1}{2}\right)$.

The rest follows easily, for a section $V=f \xi$ :

$$
\begin{aligned}
(I(V), V) & =\int_{\mathbb{S}^{l}(1 / 2) \times \mathbb{S}^{l}(1 / 2)}|\Delta V|^{2}-2 m\langle\Delta V, V\rangle v_{g} \\
& =\int_{\mathbb{S}^{l}(1 / 2) \times \mathbb{S}^{l}(1 / 2)}(\Delta f+4 l f)^{2}+8|\operatorname{grad} f|^{2}-4 l\left(f(\Delta f)+4 l f^{2}\right) v_{g} \\
& =\int_{\mathbb{S}^{l}(1 / 2) \times \mathbb{S}^{l}(1 / 2)}(\Delta f)^{2}+8 l f \Delta f+16 l^{2} f^{2}+8|\operatorname{grad} f|^{2}-4 l f(\Delta f)-16 l^{2} f^{2} v_{g} \\
& =\int_{\mathbb{S}^{l}(1 / 2) \times \mathbb{S}^{l}(1 / 2)}(\Delta f)^{2}+4 l f(\Delta f)+8|\operatorname{grad} f|^{2} v_{g} \\
& =\int_{\mathbb{S}^{l}(1 / 2) \times \mathbb{S}^{l}(1 / 2)}(\Delta f)^{2}+4(l+2)|\operatorname{grad} f|^{2} v_{g} .
\end{aligned}
$$

So, for the generalised Clifford torus, $(I(V), V)$ is always positive on the vertical sub-bundle.

Example 4.4 (Totally geodesic inclusion). Note that, though the normal bundle of $\mathbb{S}^{m}(1 / \sqrt{2})$ in $\mathbb{S}^{n}(1 / \sqrt{2})$ is not of codimension one, it is parallelized by $\left\{e_{m+2}, \ldots, e_{n+1}\right\}$ and we can compute its vertical index.

Let $V=f e_{m+i}(2 \leqslant i \leqslant n-m), f \in C^{\infty}\left(\mathbb{S}^{m}(1 / \sqrt{2})\right)$. Then $\langle V, d \phi(X)\rangle=0, \forall X \in C(T M)$ and $\langle V, \eta\rangle=0$. A short computation shows that $\Delta V=(\Delta f) e_{m+i}$.

Assuming that $\Delta f=\lambda f$ we have

$$
\begin{aligned}
(I(V), V) & =\int_{\mathbb{S}^{m}(1 / \sqrt{2})}(\langle\Delta V, \Delta V\rangle-2 m\langle\Delta V, V\rangle) v_{g} \\
& =\lambda(\lambda-2 m) \int_{\mathbb{S}^{m}(1 / \sqrt{2})} f^{2} v_{g} \\
& \geqslant 0 .
\end{aligned}
$$

Thus, vector fields $f e_{m+i}(\Delta f=\lambda f)$ do not contribute to the index of $\phi: \mathbb{S}^{m}(1 / \sqrt{2}) \rightarrow \mathbb{S}^{n+1}$. 


\section{THE INDEX OF BIHARMONIC MAPS IN SPHERES}

\section{The index}

For some maps, the study of the three different sub-bundles of the pull-back leads to an estimation of the index.

Proposition 5.1. The index of the non-harmonic biharmonic map $\mathbb{S}^{m}(1 / \sqrt{2}) \rightarrow \mathbb{S}^{n+1}$, with $m \leqslant n$, obtained from the totally geodesic inclusion $\mathbb{S}^{m}(1 / \sqrt{2}) \rightarrow \mathbb{S}^{n}(1 / \sqrt{2})$, is 1. In particular, the inclusion $\operatorname{map} \mathbb{S}^{n}(1 / \sqrt{2}) \rightarrow \mathbb{S}^{n+1}$ has index 1 .

Remark 5.2. It is interesting to compare the result of Proposition 5.1 with the index of the totally geodesic embedding $\mathbb{S}^{m}(1 / \sqrt{2}) \rightarrow \mathbb{S}^{n}(1 / \sqrt{2})$ as a harmonic map, which is $n+1$ if $m \geqslant 3$ and $n-2$ for $m=2$.

Proof of Proposition 5.1. One can easily verify that $(I(V), W)=0$ when $V$ and $W$ are orthogonal vector fields of any of the three cases, except for $V=d \phi(X)$ and $W=d \phi(Y)$, with $\operatorname{div} X=$ $\operatorname{div} Y=0$, and when $V=f \eta$ and $W=d \phi(\operatorname{grad} f)(\Delta f=\lambda f$, as usual $)$.

In the first situation, a linear combination of $V$ and $W$ can be written as $d \phi(Z)$ with $\operatorname{div} Z=0$, and therefore $\operatorname{span}\{V, W\}$ does not influence the index.

In the second case, a simple computation leads to

$$
(I(V), W)=-4 \lambda(\lambda+2-2 m) \int_{\mathbb{S}^{m}(1 / \sqrt{2})} f^{2} v_{g}
$$

and, since $(I(V), V)$ and $(I(W), W)$ are already known (and positive), we have

$$
(I(V), W)^{2} \leqslant(I(V), V)(I(W), W)
$$

for any eigenvalue $\lambda$ and dimension $m$, so that the second variation operator is positive on the span of $V$ and $W$.

The index of $\mathbb{S}^{m}(1 / \sqrt{2}) \rightarrow \mathbb{S}^{n+1}$ is therefore the sum of the different contributions examined in Examples 2.9, 3.13 and 4.4.

Proposition 5.3. The nullity of the non-harmonic biharmonic map $\mathbb{S}^{m}(1 / \sqrt{2}) \rightarrow \mathbb{S}^{n+1}$, with $m \leqslant n$, is $\frac{1}{2}(m+1)(m+2)+(m+2)(n-m)$.

Proof. A direct use of the previous computations shows that the operator $I$ preserves the following subspaces:

(i) $S_{1}=\left\{f \eta: f \in C^{\infty}\left(\mathbb{S}^{m}(1 / \sqrt{2})\right)\right\} \oplus\left\{d \phi(\operatorname{grad} g): g \in C^{\infty}\left(\mathbb{S}^{m}(1 / \sqrt{2})\right)\right\}$,

(ii) $S_{2}=\{d \phi(X): \operatorname{div} X=0\}$,

(iii) $S_{3}=\left\{f_{1} e_{m+2}+\cdots+f_{n-m} e_{n+1}: f_{1}, \ldots, f_{n-m} \in C^{\infty}\left(\mathbb{S}^{m}(1 / \sqrt{2})\right)\right\}$.

For the first space, one can easily check that, if $f$ is an eigenfunction, $I(2 f \eta+d \phi(\operatorname{grad} f))=0$ if and only if $f$ corresponds to the first non-zero eigenvalue $\lambda_{1}=2 m$ (which has multiplicity $m+1)$. Moreover, any vector field of $S_{1}$ can be written $V=\sum_{i \in \mathbb{N}} 2 \alpha_{i} f_{i} \eta+\beta_{i} d \phi\left(\operatorname{grad} f_{i}\right)$, with $f_{i}$ eigenfunction corresponding to $\lambda_{i}$.

If $I(V)=0$, then, by previous remarks, $I\left(2 \alpha_{i} f_{i} \eta+\beta_{i} d \phi\left(\operatorname{grad} f_{i}\right)\right)=0, \forall i \in \mathbb{N}$, and, necessarily, $V=\alpha_{1}\left(2 f_{1} \eta+d \phi\left(\operatorname{grad} f_{1}\right)\right)$, where $\Delta f_{1}=\lambda_{1} f_{1}$. The contribution of $S_{1}$ to the nullity is $m+1$.

Remark 3.4 shows that the space of Killing vector fields on $\mathbb{S}^{m}(1 / \sqrt{2})$, which has dimension $m(m+1) / 2$, is the kernel of $I$ restricted to $S_{2}$.

Finally, for a vertical vector field $V=f_{1} e_{m+2}+\cdots+f_{n-m} e_{n+1}$, since $\Delta V=\Delta\left(f_{1}\right) e_{m+2}+\cdots+$ $\Delta\left(f_{n-m}\right) e_{n+1}$, we see that $I(V)=0$ implies $\Delta \Delta f_{i}-2 m \Delta f_{i}=0, \forall i=1, \ldots, n-m$, so that each $f_{i}$ is a linear combination of a constant function and eigenfunctions of the first eigenvalue. Consequently, on the vertical sub-bundle, the nullity is $(m+2)(n-m)$. 


\section{E. Loubeau and C. Oniciuc}

However, in general, one cannot hope to have a parallelizable vertical sub-bundle and, having to forgo some of the sections, our results can only be lower bounds.

Proposition 5.4. The biharmonic map derived from the generalised Veronese map $\mathbb{S}^{m}(\sqrt{2(m+1) / m}) \rightarrow \mathbb{S}^{m+p}(p=(m-1)(m+2) / 2)$ has index greater than or equal to $m+2$ if $m \leqslant 4$ and $2 m+3$ if $m \geqslant 5$.

Proof. From the study of Example 2.4, we know that the index of the biharmonic map derived from the generalised Veronese map is at least $m+2$. As we know that $(I(V), V)$ is also negative when $V=d \phi(\operatorname{grad} f)$ and $m \geqslant 5$, with $\Delta f=\lambda_{1} f$ (and no other eigenvalue), a better lower bound of the index will be obtained if these vector fields combine to span an even larger space on which $(I(V), V)$ is negative definite.

This requires computing $(I(V), W)$ in two different cases: first for $V=f \eta, W=d \phi(\operatorname{grad} g)$, $\Delta f=\lambda f\left(\lambda=\lambda_{0}\right.$ or $\left.\lambda_{1}\right)$ and $\Delta g=\lambda_{1} g$; and then for $V=f \eta$ and $W=d \phi(\operatorname{grad} f), \Delta f=\lambda_{1} f$.

If $V=f \eta$ and $W=d \phi(\operatorname{grad} g), \Delta f=\lambda f, \lambda=\lambda_{0}$ or $\lambda_{1}$, and $\Delta g=\lambda_{1} g(f \neq g)$, then

$$
(I(V), W)=0
$$

since $\int_{\mathbb{S}^{m}}(\sqrt{(m+1) / m}) f g v_{g}=0$.

If $f=g$, then

$$
(I(V), W)=\frac{-4 m^{3}}{(m+1)^{2}} \int_{\mathbb{S} m(\sqrt{(m+1) / m})} f^{2} v_{g}
$$

and $(I(V), W)^{2}<(I(V), V)(I(W), W)$, so the bilinear form $(I(V), V)$ is negative definite on the space spanned by $f \eta$ and $d \phi(\operatorname{grad} f)$.

Together, the vector fields $\left\{\eta, f \eta, d \phi(\operatorname{grad} g), \Delta f=\lambda_{1} f, \Delta g=\lambda_{1} g\right\}$ (the multiplicity of $\lambda_{1}$ being $m+1)$ span a $(2 m+3)$-dimensional space on which $(I(V), V)$ is negative definite.

Various attempts to find vector fields, other than $\eta$, for which $(I(V), V)$ is negative has led us to the following conjecture.

Conjecture 5.5. The biharmonic map from $\mathbb{S}^{l}\left(\frac{1}{2}\right) \times \mathbb{S}^{l}\left(\frac{1}{2}\right)$ into $\mathbb{S}^{2 l+2}$, constructed from the generalised Clifford torus, has index 1.

\section{REFERENCES}

CMO01 R. Caddeo, S. Montaldo and C. Oniciuc, Biharmonic submanifolds of $\mathbb{S}^{3}$, Internat. J. Math. 12 (2001), 867-876.

CMO02 R. Caddeo, S. Montaldo and C. Oniciuc, Biharmonic submanifolds in spheres, Israel J. Math. 130 (2002), 109-123.

CMP01 R. Caddeo, S. Montaldo and P. Piu, Biharmonic curves on a surface, Rend. Mat. Appl. (7) 21 (2001), 143-157.

CdCK70 S. S. Chern, M. do Carmo and S. Kobayashi, Minimal submanifolds of a sphere with second fundamental form of constant length, in Functional Analysis and Related Fields, Chicago, 1968 (Springer, New York, 1970), 59-75.

EL92 J. Eells and L. Lemaire, Some properties of exponentially harmonic maps, in Partial differential equations, Banach Center Publications, vol. 27 (Polish Acad. Sci., Warsaw, 1992), 129-136.

ER93 J. Eells and A. Ratto, Harmonic maps and minimal immersions with symmetries, Annals of Mathematics Studies, vol. 130 (Princeton University Press, Princeton, NJ, 1993).

ES64 J. Eells and J. H. Sampson, Harmonic mappings of Riemannian manifolds, Amer. J. Math. 86 (1964), 109-160. 


\section{THE INDEX OF BIHARMONIC MAPS IN SPHERES}

ES66 J. Eells and J. H. Sampson, Variational theory in fibre bundles, in Proceedings of the United States-Japan seminar in differential geometry, Kyoto, Japan, 1965 (Nippon Hyoronsha, Tokyo, 1966), 22-33.

EW76 J. Eells and J. C. Wood, Restrictions on harmonic maps of surfaces, Topology 15 (1976), 263-266.

Jia86 G. Y. Jiang, 2-harmonic maps and their first and second variational formulas, Chinese Ann. Math. Ser. A 7 (1986), 389-402.

Leu82 P. F. Leung, On the stability of harmonic maps, in Harmonic maps, Tulane 1980, Lecture Notes in Mathematics, vol. 949 (Springer, New York, 1982), 122-129.

Lic58 A. Lichnerowicz, Géométrie des groupes de transformations (Dunod, Paris, 1958).

Oni02 C. Oniciuc, On the second variation formula for biharmonic maps to a sphere, Publ. Math. Debrecen 61 (2002), 613-622.

Smi75 R. T. Smith, The second variation formula for harmonic mappings, Proc. Amer. Math. Soc. 47 (1975), 229-236.

Yan52 K. Yano, On harmonic and Killing vector fields, Ann. of Math. (2) 55 (1952), 38-45.

E. Loubeau loubeau@univ-brest.fr

Département de Mathématiques, Laboratoire CNRS UMR 6205, Université de Bretagne Occidentale, 6 avenue Victor Le Gorgeu, CS 93837, 29238 Brest cedex 3, France

C. Oniciuc oniciucc@uaic.ro

Faculty of Mathematics, Al. I. Cuza University of Iaşi, Bd. Copou Nr. 11, 6600 Iaşi, Romania

Current address: Département de Mathématiques, Laboratoire CNRS UMR 6205, Université de Bretagne Occidentale, 6 avenue Victor Le Gorgeu, CS 93837, 29238 Brest cedex 3, France 